\title{
Discrete-time integral MRAC with minimal controller synthesis and parameter projection
}

\author{
Umberto Montanaro* \& Josep M. Olm ${ }^{\dagger}$
}

\begin{abstract}
Model reference adaptive controllers with Minimal Control Synthesis are effective control algorithms to guarantee asymptotic convergence of the tracking error to zero not only for disturbance-free uncertain linear systems, but also for highly nonlinear plants with unknown parameters, unmodeled dynamics and subject to perturbations. However, an apparent drift in adaptive gains may occasionally arise, which can eventually lead to closed-loop instability. In this article, we address this key issue for discrete-time systems under $L_{2}$ disturbances using a parameter projection algorithm. A consistent proof of stability of all the closed-loop signals is provided, while tracking error is shown to asymptotically converge to zero. We also show the applicability of the adaptive algorithm for digitally controlled continuous-time plants. The proposed algorithm is numerically validated taking into account a discrete-time LTI system subject to parameter uncertainty, parameter variations and $L_{2}$ disturbances. Finally, as a possible engineering application of this novel adaptive strategy, the control of a highly nonlinear electromechanical actuator is considered.
\end{abstract}

\section{Introduction}

Adaptive control algorithms are particularly useful in a wide range of applications, specifically those affected by noise and uncertainties. Hence, both researchers and practitioners have been interested in expanding adaptive control theory and

\footnotetext{
*Department of Industrial Engineering, University of Naples Federico II, Naples 80125, Italy

${ }^{\dagger}$ Department of Mathematics \& Institute of Industrial and Control Engineering, Universitat Politècnica de Catalunya, 08028 Barcelona, Spain
} 
using the best adaptive schemes available in literature. The reader is referred to the classical textbooks [23, 41] for an overview of adaptive control techniques. Notwithstanding, this is also an active field of research: for example, and just to name a few recent works, in [29] a modular output based variable structure adaptive backstepping strategy for systems with arbitrary relative degrees has been designed, while in [43] an adaptive control approach for a class of uncertain nonlinear systems with unknown time-varying delays in the pure-feedback form has been proposed. In addition, in [6] an adaptive compensation control design is developed via disturbance observer and quantum information technology for a four-rotor helicopter.

The Model Reference Adaptive Control (MRAC) algorithm with Minimal Controller Synthesis (MCS) $[35,36]$ is an adaptive control scheme that belongs to the family of passivity-based MRAC controllers introduced in [24]. The main feature of the MCS approach, which demands the system to be linear and in control canonical form, is the minimal amount of a-priori information needed from the plant in order to devise the controller, as it just requires knowledge of the sign of the non-null matrix element of the input vector. Indeed, the MCS algorithm is effective in the control of plants with unknown parameters, and provides robustness to parameter variations.

Since its appearance, MCS controllers have been used in a number of applications as, for example, synchronization of chaotic systems [39], shaking tables in civil engineering [40], active engine mounts [22], hydraulic test rigs [21], cantilever beams [31], electronic throttle valves [11, 9], common rail systems [26, 25] and electromechanical valve actuators for future camless engines [17]. Moreover, the theoretical body of the MCS has been enhanced with several extensions to the original algorithms for continuous-time and discrete-time systems. Among the first ones are the decentralized MCS [4], the extended MCS [38] -which includes a switching action to cope with rapidly varying disturbances-, and the Integral MCS (MCSI) [37] -with an explicit integral action to make the control effort independent of the operation set point and to prevent possible gain wind-up due to plant disturbances and signal offsets-. More recently, novel MCS strategies have been proposed also for controlling Piecewise Affine (PWA) systems [14, 15], which have been experimentally validated in [16].

A Discrete-Time counterpart of the MCS (DTMCS), including a formal stability proof, was proposed in [12], experimentally validated in [8], and extended to piecewise linear systems in [13]. Recently, discrete-time MCS algorithms including integral action (DTMCSI) and integral plus switching action have been proposed in [26, 27], respectively. 
Despite its proved effectiveness in imposing the dynamics of a preassigned reference model to a given plant, the MCS algorithm suffers from a severe drawback which is common to similar MRAC controllers. Indeed, although theoretical results guarantee global asymptotic stability of the tracking error to zero, boundedness of all closed-loop signals is only ensured for uncertain Linear Time Invariant (LTI) systems. In more detail, an unbounded drift of adaptive gains may arise when the plant is affected by unmodeled dynamics or disturbances even though they are matched (see, for example, $[2,41]$ and references therein). Such a behavior is occasionally encountered by MCS practitioners, as for example in two relevant plants in the context of the automotive engineering, i.e., the Electronic Throttle Body and the Common Rail $[8,26]$. However, neither the MCSI algorithm nor the method proposed in [34] solve the gain locking problem systematically, and only slower gain drifting can be achieved.

Among the different methods available in the MRAC literature to ensure robustness in the face of unmodeled dynamics or disturbances (see, for example, [41], and references therein), parameter projection has been often adopted. For example, a full state parameter projection-based adaptive law has been used in $[32,33]$ to address the MRAC adaptive control problem in continuous-time piecewise linear systems. Inspired by the continuous-time case, in this paper we propose an analytical solution to cope with the possible onset of adaptive gains instability for discrete-time MCS strategies.

Specifically, we enhance the Discrete-Time MCS strategy with Integral action (DTMCSI) proposed in [26] by including a Parameter Projection (PP)-based gain locking method. The control aim is to solve the MRAC control problem for discrete-time LTI systems when plant parameters are affected by uncertainties and plant dynamics are subjected to an additive, square-integrable unknown disturbance, while ensuring boundedness of adaptive gains in a given set.

The novel control action, termed with the acronym DTMCSI-PP, can be applied also to Linear Time-Varying (LTV) systems provided that the plant parameters variation is slower than that of the adaptive gains. This assumption is often made in the MCS literature when deriving MCS laws for both continuous-time and discrete-time LTV systems [36, 12]. Moreover, as happens with the discrete-time MCS [12], the DTMCSI-PP here derived can be used to control continuous-time systems when the forward Euler method is used for their discretization.

The effectiveness of the DTMCSI-PP strategy is shown on a set of representative numerical examples. In particular, we first consider the control of a discrete-time LTI system affected by uncertain plant parameters, $L_{2}$-disturbances, and plant parameter variations. Then, as a realistic case study, we consider the 
control of a highly nonlinear actuator, namely an Electronic Throttle Body. The obtained results confirm the theoretical findings, and excellent tracking performances are achieved. Indeed, it is shown that the DTMCSI-PP performs better than the continuous-time MCSI [37] discretized with a Tustin method. Moreover, the novel algorithm yields residual errors that are comparable to the DTMCSI strategy [26], but with the adaptive gains always evolving in a bounded preassigned set.

\section{Discrete-time integral MCS with parameter pro- jection}

Consider a plant of the form

$$
x(k+1)=A x(k)+B_{p} u(k)+B d(k), \quad x(0) \in \mathbb{R}^{n},
$$

where $x \in \mathbb{R}^{n}$ and $u \in \mathbb{R}$ are the state and the input of the system, respectively, and $d \in L_{2}$ is a possible disturbance acting on the plant dynamics, with $n \in \mathbb{N}$ being the dimension of the state space, while the system matrices $A \in \mathbb{R}^{n \times n}$, $B_{p} \in \mathbb{R}^{n \times 1}$ and $B \in \mathbb{R}^{n \times 1}$ are in control canonical form, i.e.

$$
\begin{aligned}
A & =\left[\begin{array}{ccccc}
0 & 1 & 0 & \ldots & 0 \\
0 & 0 & 1 & \ldots & 0 \\
\vdots & \vdots & \vdots & \ddots & \vdots \\
0 & 0 & 0 & \ldots & 1 \\
-a_{1} & -a_{2} & \ldots & \ldots & -a_{n}
\end{array}\right], \\
B_{p} & =\left[\begin{array}{lllll}
0 & 0 & \ldots & 0 & b
\end{array}\right]^{T}=b B \\
B & =\left[\begin{array}{lllll}
0 & 0 & \ldots & 0 & 1
\end{array}\right]^{T}
\end{aligned}
$$

The following hypotheses are assumed for the plant parameters.

\section{Assumption A}

(i) Upper and lower bounds are known for the plant parameters $a_{i}$, i.e., $a_{i} \in$ $\left[a_{i}^{l}, a_{i}^{u}\right]$, with $a_{i}^{l} \leq a_{i}^{u}$ known constants, $\forall i=1, \ldots, n$.

(ii) The gain parameter $b$ has definite sign, and upper and lower bounds are known for it as well, i.e. $b \in\left[b_{i}^{l}, b_{i}^{u}\right]$, with $b_{i}^{l} \leq b_{i}^{u}$ known, and either $b_{i}^{l}>0$ or $b_{i}^{u}<0$.

The target is to devise a control law to impose to system (1) the dynamics of a certain asymptotically stable reference model while keeping all the closed-loop 
signals bounded. The reference model is assumed to be a linear time-invariant system of the form

$$
x_{m}(k+1)=A_{m} x_{m}(k)+B_{m} r(k), \quad x_{m}(0) \in \mathbb{R}^{n},
$$

where $x_{m} \in \mathbb{R}^{n}$ and $r(k) \in \mathbb{R}$ are the state and the input of the reference model, respectively, and $A_{m}, B_{m}$ have the same dimensions and canonical form as those of the plant matrices $A, B$, respectively, i.e.

$$
\begin{aligned}
A_{m} & =\left[\begin{array}{ccccc}
0 & 1 & 0 & \ldots & 0 \\
0 & 0 & 1 & \ldots & 0 \\
\vdots & \vdots & \vdots & \ddots & \vdots \\
0 & 0 & 0 & \ldots & 1 \\
-a_{m_{1}} & -a_{m_{2}} & \ldots & \ldots & -a_{m_{n}}
\end{array}\right], \\
B_{m} & =\left[\begin{array}{lllll}
0 & 0 & \ldots & 0 & b_{m}
\end{array}\right]^{T}=b_{m} B
\end{aligned}
$$

Moreover, the following design assumptions are made for the reference model.

\section{Assumption B}

(i) $A_{m}$ is a discrete-time Hurwitz matrix.

(ii) The reference input is bounded, i.e. $r \in L_{\infty}$.

(iii) Let $\mathbb{I}$ denote the unit matrix in $\mathbb{R}^{n \times n}$. Then $Q=Q^{T}$ is a symmetric matrix so that $Q-\mathbb{I}>0$, while $P=P^{T}>0$ is a solution of the discrete-time Lyapunov equation

$$
A_{m}^{T} P A_{m}-P=-Q
$$

The DTMCSI-PP algorithm to solve the MRAC problem, i.e. $x \rightarrow x_{m}$ as $k \rightarrow+\infty$, is:

$$
u(k)=u_{M C S}(k)+u_{E}(k),
$$

with

$$
\begin{aligned}
u_{M C S}(k) & =L_{X}(k) x(k)+L_{R}(k) r(k), \\
u_{E}(k) & =L_{E}(k) x_{E}(k),
\end{aligned}
$$

where

$$
x_{E}(k)=\sum_{i=0}^{k} x_{e}(i), \quad x_{e}(k)=x_{m}(k)-x(k),
$$


while the adaptive control gains are computed as

$$
\begin{aligned}
& L_{X}(k)=L_{X I}(k)+\beta y_{e}(k+1) x^{T}(k), \\
& L_{R}(k)=L_{R I}(k)+\beta y_{e}(k+1) r(k), \\
& L_{E}(k)=L_{E I}(k)+\beta_{E} y_{e}(k+1) x_{E}^{T}(k),
\end{aligned}
$$

with $y_{e}$ being an auxiliary output selected as

$$
y_{e}(k)=B^{T} P x_{e}(k) \in \mathbb{R} .
$$

The integral parts of the gains in (9) are computed as

$$
\begin{aligned}
L_{X I}(k) & =\widetilde{L}_{X I}(k)+f_{X}(k), \\
L_{R I}(k) & =\widetilde{L}_{R I}(k)+f_{R}(k), \\
L_{E I}(k) & =\widetilde{L}_{E I}(k)+f_{E}(k),
\end{aligned}
$$

where the functions $\widetilde{L}_{X I}, \widetilde{L}_{R I}, \widetilde{L}_{E I}$ are defined as

$$
\begin{aligned}
& \widetilde{L}_{X I}(k)=L_{X I}(k-1)+\alpha y_{e}(k) x^{T}(k-1), \\
& \widetilde{L}_{R I}(k)=L_{R I}(k-1)+\alpha y_{e}(k) r(k-1), \\
& \widetilde{L}_{E I}(k)=L_{E I}(k-1)+\alpha_{E} y_{e}(k) x_{I}^{T}(k-1),
\end{aligned}
$$

while $f_{X}(k) \in \mathbb{R}^{n}, f_{R}(k) \in \mathbb{R}$ and $f_{E}(k) \in \mathbb{R}^{n}$ are defined componentwise as

$$
\begin{aligned}
& f_{X_{j}}=\left\{\begin{array}{ccc}
0 & \text { if } & \widetilde{L}_{X I_{j}}(k) \in\left[L_{X I_{j}}^{l}, L_{X I_{j}}^{u}\right] \\
L_{X I_{j}}^{u}-\widetilde{L}_{X I_{j}}(k) \text { if } & \widetilde{L}_{X I_{j}}(k)>L_{X I_{j}}^{u} \\
L_{X I_{j}}^{l}-\widetilde{L}_{X I_{j}}(k) \text { if } & \widetilde{L}_{X I_{j}}(k)<L_{X I_{j}}^{l},
\end{array}\right. \\
& f_{R}=\left\{\begin{array}{ccc}
0 & \text { if } & \widetilde{L}_{R I}(k) \in\left[L_{R I}^{l}, L_{R I_{j}}^{u}\right] \\
L_{R I}^{u}-\widetilde{L}_{R I}(k) \text { if } & \widetilde{L}_{R I}(k)>L_{R I}^{u} \\
L_{R I}^{l}-\widetilde{L}_{R I}(k) \text { if } & \widetilde{L}_{R I}(k)<L_{R I}^{l},
\end{array}\right. \\
& f_{E_{j}}=\left\{\begin{array}{ccc}
0 & \text { if } & \widetilde{L}_{E I_{j}}(k) \in\left[L_{E I_{j}}^{l}, L_{E I_{j}}^{u}\right] \\
L_{E I_{j}}^{u}-\widetilde{L}_{E I_{j}}(k) \text { if } & \widetilde{L}_{E I_{j}}(k)>L_{E I_{j}}^{u} \\
L_{E I_{j}}^{l}-\widetilde{L}_{E I_{j}}(k) \text { if } & \widetilde{L}_{E I_{j}}(k)<L_{E I_{j}}^{l} .
\end{array}\right.
\end{aligned}
$$

The control weights and the bounds of the integral parts of the control gains are chosen as follows. 


\section{Assumption C}

(i) The control weights $\alpha, \alpha_{E}, \beta, \beta_{E}$ are so that:

$$
\begin{aligned}
& \operatorname{sign}(\alpha)=\operatorname{sign}(\beta)=\operatorname{sign}(b), \\
& \operatorname{sign}\left(\alpha_{E}\right), \operatorname{sign}\left(\beta_{E}\right) \in\{0, \operatorname{sign}(b)\}, \\
& |\beta| \geq \frac{|\alpha|}{2}, \quad\left|\beta_{E}\right| \geq \frac{\left|\alpha_{E}\right|}{2} .
\end{aligned}
$$

(ii) The integral parts of the control gains $L_{X I_{j}}^{l}, L_{X I_{j}}^{u}, L_{E I_{j}}^{l}, L_{E I_{j}}^{u}, j=1, \ldots, n$, and $L_{R I}^{l}, L_{R I}^{u}$, are chosen so as to verify:

$$
\frac{a_{m_{j}}-a_{j}}{b} \in\left[L_{X I_{j}}^{l}, L_{X I_{j}}^{u}\right], 0 \in\left[L_{E I_{j}}^{l}, L_{E I_{j}}^{u}\right], \frac{b_{m}}{b} \in\left[L_{R I}^{l}, L_{R I}^{u}\right] .
$$

Remark 1. Assumption C.i can be fulfilled when A.ii is verified. In addition, as the model reference parameters $a_{m_{j}}$ and $b_{m}$ are known by design, the parameters $L_{X I_{j}}^{l}, L_{X I_{j}}^{u}, L_{R I}^{l}, L_{R I}^{u}$ in Assumption C.ii can be readily obtained from $a_{j}^{l}, a_{j}^{u}, b_{j}^{l}, b_{j}^{u}$ when Assumption A is verified.

The control goal, namely, convergence of the tracking error to zero and boundedness of all the closed-loop signals under square-summable disturbances, is guaranteed by the following result:

Theorem 1. Consider system (1) and the reference model (3). If Assumptions A, $B$ and $C$ are fulfilled, for all $j=1 \ldots n$, then

$$
L_{X I_{j}}(0) \in\left[L_{X I_{j}}^{l}, L_{X I_{j}}^{u}\right], L_{R I}(0) \in\left[L_{R I}^{l}, L_{R I}^{u}\right], L_{E I_{j}}(0) \in\left[L_{E I_{j}}^{l}, L_{E I_{j}}^{u}\right] .
$$

Furthermore if $d \in L_{2}$, then the adaptive control law (6)-(13) guarantees $x_{e}(k) \rightarrow$ 0 as $k \rightarrow \infty$, and $x, x_{E}, L_{X}, L_{R}, L_{E}, u \in L_{\infty}$.

\section{Remark 2.}

i) The parameter projection method consists of using the parameter projection functions (13) for the computation of the integral part of the adaptive gains (11). 
ii) In case $f_{X}, f_{R}$ and $f_{E}$ are set to zero, the control action (6)-(13) boils down to the DTMCSI presented in [26]. However, the DTMCSI algorithm gains $\widetilde{L}_{X I}(k), \widetilde{L}_{R I}(k), \widetilde{L}_{E I}(k)$ can then grow unbounded in the face of disturbances or unmodelled dynamics even though $x(k), x_{E}(k)$ and the input $r(k)$ are bounded. Hence, the novel algorithm improves and extends those available in the literature by guaranteeing an evolution of the control gains in a bounded and preassigned set. Furthermore, differently from [26], it is analytically shown here that unknown $L_{2}$ disturbances do not alter closedloop stability.

iii) In the disturbance-free case, i.e. $d(k)=0, \forall k \geq 0$, the requirement $Q-\mathbb{I}>$ 0 in (5) can be replaced by $Q>0$ as usually assumed for the DTMCS [12] and the DTMCSI [26] strategies. However, using a Q-matrix that satisfies assumption B.iii does not entail any limitation because it is selected by designers.

iv) The adaptive mechanism (9) at the discrete-time instant $t=k$ requires knowledge of the output signal at time $k+1$, namely $y_{e}(k+1)$. This problem, known as the one-delay problem in MRAC literature [24], can be practically solved by means of an estimate of the sample $y_{e}(k+1)$ such as the one proposed in [24, 12].

v) It is worth noticing that in discrete-time MCS algorithms proposed in the literature so far, the summation for the integral part of the control gains is up to the current time instant $k$, thus including part of the one-delay term, while the rest is in the proportional gain. However, in (9), (11), (12) the integral gain is not affected by the one-delay problem, as the one-delay term is entirely included in the proportional part of the adaptive gains. Hence, the one-delay issue has to be considered only when manipulating the proportional adaptive terms, thus simplifying the proof of Theorem 1 using a Lyapunov approach.

vi) From the definition of the updating mechanism (11), it is evident that the in-

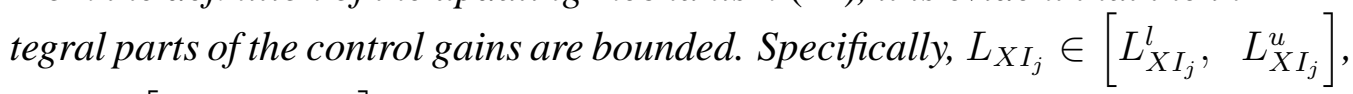
$L_{E I_{j}} \in\left[L_{E I_{j}}^{l}, L_{E I_{j}}^{u}\right], j=1,2, \ldots, n$, and $L_{R I} \in\left[\begin{array}{ll}L_{R I}^{l}, & L_{R I}^{u}\end{array}\right]$.

vii) The only extra assumption that is required with respect to the MCS strategy presented in [26] is that some bounds for the plant parameters are known. 
This is not a limitation, because nominal values for the plant parameters, as well as their range of variations, are often available in engineering problems.

The result of Theorem 1 still holds for LTV plants under the assumption of slow variation of the plant parameters with respect to the integral part of the adaptation gains according to the following corollary. It is worth stressing that this is a standard assumption in the MCS literature both for continuous-time and discretetime systems $[36,12]$.

Corollary 1. Let the plant defined in (2a) be time-varying, i.e. $A=A(k)$ with $a_{i}=a_{i}(k)$ in the last row of $A(k)$. If, for all $i=1, \ldots, n$,

$$
a_{i}(k-1)-a_{i}(k)-\alpha b y_{e}(k) x_{i}(k-1) \approx-\alpha b y_{e}(k) x_{i}(k-1),
$$

then the adaptive control law (6) yields convergence of the tracking error to zero, and boundedness of all closed-loop signals.

Finally, as happens with the discrete-time MCS in [12, 26], also the DTMCSIPP algorithm can be applied to control continuous-time plants in control canonical form when both the plant dynamics and that of the reference model are discretized by using a forward Euler discretization method. Indeed, consider the continuoustime linear system and reference model

$$
\begin{aligned}
\dot{x} & =A x+B_{p} u+B d(t), \\
\dot{x}_{m} & =A_{m} x_{m}+B_{m} r(t),
\end{aligned}
$$

where $x, x_{m}, \in \mathbb{R}^{n}, u, d, r \in \mathbb{R}, A, A_{m} \in \mathbb{R}^{n \times n}, B_{p}, B_{m}, B \in \mathbb{R}^{n \times 1}, n \in \mathbb{N}$ and $A, B_{p}, B, A_{m}, B_{m}$ are defined in (2), (4). A forward Euler discretization of (17)-(18) with sampling period $T_{s} \in \mathbb{R}^{+}$, yields:

$$
\begin{aligned}
x(k+1) & =\left(\mathbb{I}+T_{s} A\right) x(k)+T_{s} B_{p} u(k)+T_{s} B d(k), \\
x_{m}(k+1) & =\left(\mathbb{I}+T_{s} A_{m}\right) x_{m}(k)+T_{s} B_{m} r(k),
\end{aligned}
$$

and the following corollary holds.

Corollary 2. Consider system (19) and the reference model (20). Let Assumptions $A, B$, and $C$ be met, with $\mathbb{I}+T_{s} A_{m}$ replacing $A_{m}$ in Assumption B.i and (5), and $T_{s} b^{-1}\left(a_{m_{j}}-a_{j}\right), T_{s} b^{-1} b_{m}$ replacing $b^{-1}\left(a_{m_{j}}-a_{j}\right), b^{-1} b_{m}$, respectively, in Assumption C.ii. Let also the integral part of the control gains be initialized as in (15). If $d \in L_{2}$, then the adaptive control law (6)-(13) yields $x(k) \rightarrow x_{m}(k)$ as $k \rightarrow \infty$, and $x, x_{E}, L_{X}, L_{R}, L_{E}, u \in L_{\infty}$. 
We note that, even though the forward Euler method does not guarantee stability of the discretized plant, this is not restrictive since the DTMCSI-PP strategy can be applied also to unstable plants, and the stability of the discretized reference model can always be assured as it is chosen by the designer. Moreover, the use of such a digital strategy prevents the onset of unwanted phenomena -instability and undesirable overshoots- that can arise when implementing continuous-time MCS algorithms via standard discretization methods [5].

\section{Proof of the main results}

The proof of is derived by using Lyapunov theory for discrete-time systems. Indeed, while passivity/hyperstability theory is often used to prove tracking error convergence in Landau's schemes-based discrete time MRAC strategies, the Lyapunov approach simplifies proofs when parameter projection methods are used to ensure boundedness of adaptive gains.

\subsection{Proof of Theorem 1}

As in [24], the control law (6)-(13) can be written as an integral-like term plus a proportional-like term. Namely,

$$
u(k)=L(k) w(k)=\left(L_{I}(k)+L_{P}(k)\right) w(k),
$$

with

$$
\begin{gathered}
L_{I}(k)=L_{I}(k-1)+y_{e}(k) w^{T}(k-1) \Gamma_{\alpha}+f(k), \\
L_{P}(k)=y_{e}(k+1) w^{T}(k) \Gamma_{\beta}, \\
L_{I}^{T}(k)=\left[\begin{array}{c}
L_{X I}(k) \\
L_{R I}(k) \\
L_{E I}(k)
\end{array}\right], \quad w(k)=\left[\begin{array}{c}
x(k) \\
r(k) \\
x_{E}(k)
\end{array}\right], \quad f^{T}(k)=\left[\begin{array}{c}
f_{X}(k) \\
f_{R}(k) \\
f_{E}(k)
\end{array}\right],
\end{gathered}
$$

and

$$
\Gamma_{\alpha, \beta}=\operatorname{diag}\left(\gamma_{1}^{\alpha, \beta}, \ldots, \gamma_{2 n+1}^{\alpha, \beta}\right),
$$

where $\left(\gamma_{i}^{\alpha}, \gamma_{i}^{\beta}\right)=(\alpha, \beta), i=1, \ldots, n+1$, and $\left(\gamma_{i}^{\alpha}, \gamma_{i}^{\beta}\right)=\left(\alpha_{E}, \beta_{E}\right), i=$ $n+2, \ldots, 2 n+1$. 
Let us define

$$
\begin{aligned}
\Phi(k+1) & =\phi(k) w(k)-b L_{P}(k) w(k) \\
\phi(k) & =b\left(L_{I}^{*}-L_{I}(k)\right) \\
L_{I}^{*} & =b^{-1}\left(a_{m, 1}-a_{1}, \ldots, a_{m, n}-a_{n}, \mathbb{O}_{1, n}, b_{m}\right),
\end{aligned}
$$

which allows to obtain

$$
\Delta \phi(k-1)=\phi(k)-\phi(k-1)=-b y_{e}(k) w^{T}(k-1) \Gamma_{\alpha}-b f(k) .
$$

With the above settings, and recalling from (8) that $x_{e}(k)=x_{m}(k)-x(k)$, the closed-loop error dynamics associated to (2), (3), (21)-(24) can be written as

$$
\begin{aligned}
x_{e}(k+1) & =A_{m} x_{e}(k)+B \Phi(k+1)-B d(k), \\
y_{e}(k) & =B^{T} P x_{e}(k) .
\end{aligned}
$$

Now, recalling Assumption C.ii, let us define

$$
\begin{aligned}
L_{I_{i}}^{l, u} & = \begin{cases}L_{X I_{i},}^{l, u}, & i=1, \ldots, n, \\
L_{R I_{i}}^{l, u}, & i=n+1, \\
L_{E I_{i}}^{l, u}, & i=n+2, \ldots, 2 n+1,\end{cases} \\
\Lambda & =\Lambda_{1} \times \cdots \Lambda_{n}=\left[L_{I_{1}}^{l}, L_{I_{1}}^{u}\right] \times \cdots \times\left[L_{I_{2 n+1}}^{l}, L_{I_{2 n+1}}^{u}\right] .
\end{aligned}
$$

Lemma 1. Let $L_{I}(k)$ be updated as in (22a).

i) If $L_{I}(0) \in \Lambda$, then $L_{I}(k) \in \Lambda, \forall k \geq 0$.

ii) Let $L_{I}^{*} \in \Lambda$; then, $\left(L_{I, i}(k)-L_{I, i}^{*}\right) f_{i}(k) \leq 0, \forall i=1, \ldots, 2 n+1, \forall k \geq 0$.

Proof. Straightforward.

Consider the auxiliary function

$$
V(k)=V_{1}(k)+V_{2}(k),
$$

with

$$
\begin{aligned}
V_{1}(k) & =\left(x_{e}(k)-B \Phi(k)\right)^{T} P\left(x_{e}(k)-B \Phi(k)\right), \\
V_{2}(k) & =\frac{1}{b} \phi(k-1) \Gamma_{\alpha}^{-1} \phi^{T}(k-1) .
\end{aligned}
$$


The time variation of the first component of $V(k)$ is given by:

$$
\begin{aligned}
\Delta V_{1}(k) & =V_{1}(k+1)-V_{1}(k)= \\
& =\left(x_{e}(k+1)-B \Phi(k+1)\right)^{T} P\left(x_{e}(k+1)-B \Phi(k+1)\right)+ \\
& -\left(x_{e}(k)-B \Phi(k)\right)^{T} P\left(x_{e}(k)-B \Phi(k)\right) ;
\end{aligned}
$$

taking into account (26), after some algebraic manipulations, we have

$$
\begin{aligned}
\Delta V_{1}(k) & =\left(A_{m} x_{e}(k)-B d(k)\right)^{T} P\left(A_{m} x_{e}(k)-B d(k)\right)-x_{e}^{T}(k) P x_{e}(k)+ \\
& -B^{T} P B \Phi^{2}(k)+2 B^{T} P x_{e}(k) \Phi(k)= \\
& =x_{e}^{T}(k)\left(A_{m}^{T} P A_{m}-P\right) x_{e}(k)-2 B^{T} P A_{m} x_{e}(k) d(k)+B^{T} P B d^{2}(k)+ \\
& -B^{T} P B \Phi^{2}(k)+2 y_{e}(k) \Phi(k) .
\end{aligned}
$$

Now, by using the hypothesis (5) and proceeding to square $-2 B^{T} P A_{m} x_{e}(k) d(k)$ one gets

$$
\begin{aligned}
\Delta V_{1}(k) & =-x_{e}(k)^{T} Q x_{e}(k)-\left(x_{e}(k)+A_{m}^{T} P B d(k)\right)^{T}\left(x_{e}(k)+A_{m}^{T} P B d(k)\right)+ \\
& +x_{e}^{T}(k) x_{e}(k)+B^{T}\left(P A_{m} A_{m}^{T} P+P\right) B d^{2}(k)-B^{T} P B \Phi^{2}(k)+ \\
& +2 B^{T} P y_{e}(k) \Phi(k)= \\
& =-x_{e}(k)^{T}(Q-\mathbb{I}) x_{e}(k)-\left\|x_{e}(k)+A_{m}^{T} P B d(k)\right\|^{2}-B^{T} P B \Phi^{2}(k)+ \\
& +B^{T}\left(P A_{m} A_{m}^{T} P+P\right) B d^{2}(k)+2 y_{e}(k) \Phi(k),
\end{aligned}
$$

with $\|\cdot\|$ denoting the Euclidean norm of a vector.

Considering now $V_{2}(k)$, from (25) it is possible to compute its variation as

$$
\begin{aligned}
\Delta V_{2}(k) & =V_{2}(k+1)-V_{2}(k)=\frac{1}{b} \phi(k) \Gamma_{\alpha}^{-1} \phi^{T}(k)-\frac{1}{b} \phi(k-1) \Gamma_{\alpha}^{-1} \phi^{T}(k-1)= \\
& =\frac{1}{b}(\phi(k-1)+\Delta \phi(k-1)) \Gamma_{\alpha}^{-1}\left(\phi(k-1)+\frac{1}{b} \Delta \phi(k-1)\right)^{T}+ \\
& -\frac{1}{b} \phi(k-1) \Gamma_{\alpha}^{-1} \phi^{T}(k-1)= \\
& =\frac{2}{b} \Delta \phi(k-1) \Gamma_{\alpha}^{-1} \phi^{T}(k-1)+\frac{1}{b} \Delta \phi(k-1) \Gamma_{\alpha}^{-1} \Delta \phi(k-1)^{T} .
\end{aligned}
$$


Then, (25), (24a) and (22b) yield

$$
\begin{aligned}
\Delta V_{2}(k) & =-2\left(y_{e}(k) w^{T}(k-1) \Gamma_{\alpha}+f(k)\right) \Gamma_{\alpha}^{-1} \phi^{T}(k-1)+ \\
& +b\left(y_{e}(k) w^{T}(k-1) \Gamma_{\alpha}+f(k)\right) \Gamma_{\alpha}^{-1}\left(y_{e}(k) w^{T}(k-1) \Gamma_{\alpha}+f(k)\right)^{T}= \\
& =-2 y_{e}(k)\left(\Phi(k)+b L_{P}(k-1) w(k-1)\right)+ \\
& +b y_{e}^{2}(k) w^{T}(k-1) \Gamma_{\alpha} w(k-1)+ \\
& +2\left(b y_{e}(k) w^{T}(k-1)-\phi(k-1) \Gamma_{\alpha}^{-1}\right) f^{T}(k)+b f(k) \Gamma_{\alpha}^{-1} f^{T}(k)= \\
& =-2 y_{e}(k) \Phi(k)-2 b y_{e}^{2}(k) w^{T}(k-1) \Gamma_{\beta} w(k-1)+ \\
& +b y_{e}^{2}(k) w^{T}(k-1) \Gamma_{\alpha} w(k-1)+ \\
& -2(\Delta \phi(k-1)+\phi(k-1)+b f(k)) \Gamma_{\alpha}^{-1} f^{T}(k)+b f(k) \Gamma_{\alpha}^{-1} f^{T}(k) .
\end{aligned}
$$

Finally, taking into account $(24 \mathrm{~b}), \Delta V_{2}(k)$ becomes

$$
\begin{aligned}
\Delta V_{2}(k)=-2 y_{e}(k) \Phi(k) & -b y_{e}^{2}(k) w^{T}(k-1)\left(2 \Gamma_{\beta}-\Gamma_{\alpha}\right) w(k-1)+ \\
& +2 b\left(L_{I}(k)-L_{I}^{*}\right) \Gamma_{\alpha}^{-1} f^{T}(k)-b f(k) \Gamma_{\alpha}^{-1} f^{T}(k) .
\end{aligned}
$$

From (29)-(31), the variation of the function $V(k)$ in (29) is

$$
\begin{aligned}
\Delta V(k) & =-x_{e}(k)^{T}(Q-\mathbb{I}) x_{e}(k)-\left\|x_{e}(k)+A_{m}^{T} P B d(k)\right\|^{2}-B^{T} P B \Phi^{2}(k)+ \\
& -b y_{e}^{2}(k) w^{T}(k-1)\left(2 \Gamma_{\beta}-\Gamma_{\alpha}\right) w(k-1)+2 b\left(L_{I}(k)-L_{I}^{*}\right) \Gamma_{\alpha}^{-1} f^{T}(k)+ \\
& -b f(k) \Gamma_{\alpha}^{-1} f^{T}(k)+B^{T}\left(P A_{m} A_{m}^{T} P+P\right) B d^{2}(k) .
\end{aligned}
$$

As $Q-\mathbb{I}$ and $P$ are symmetric, positive definite matrices (see Assumption B.iii), $b\left(2 \Gamma_{\beta}-\Gamma_{\alpha}\right)$ and $b \Gamma_{\alpha}^{-1}$ are diagonal, positive definite matrices by the inequalities (14) in Assumption C.i, and $\left(L_{I, i}(k)-L_{I, i}^{*}\right) f_{i}(k) \leq 0, \forall i=1, \ldots, 2 n+1$ in accordance with Lemma 1, the function $\Delta V(k)$ in (32) can be bounded as

$$
\Delta V(k) \leq-\lambda_{\min }\left\|x_{e}(k)\right\|^{2}+\delta d^{2}(k),
$$

with $\lambda_{\min }>0$ being the lowest eigenvalue of $Q-\mathbb{I}$ and

$$
\delta=B^{T}\left(P A_{m} A_{m}^{T} P+P\right) B>0 .
$$

Adding up for all $k$ in (33) we get

$$
\begin{aligned}
V(+\infty)-V(0) & =\sum_{k \geq 0} \Delta V(k) \leq-\lambda_{\min } \sum_{k \geq 0}\left\|x_{e}(k)\right\|^{2}+\delta \sum_{k \geq 0} d^{2}(k)= \\
& =-\lambda_{\min }\left\|x_{e}\right\|_{2}^{2}+\delta\|d\|_{2}^{2}
\end{aligned}
$$


where $\|\cdot\|_{2}$ stands for the $L_{2}$-norm. As $V(0)$ is bounded (the initial adaptive gains, plant and reference model state are assumed to be finite), $d \in L_{2}, V$ is positive semidefinite, and $\left\|x_{e}\right\| \geq 0$, (34) implies that

$$
0 \leq V(+\infty)+\lambda_{\min }\left\|x_{e}\right\|_{2}^{2} \leq V(0)+\delta\|d\|_{2}^{2}<+\infty .
$$

Hence, $x_{e} \in L_{2} \cap L_{\infty}$, and consequently $x_{e}(k) \rightarrow 0$ for $k \rightarrow+\infty$. From Assumptions B.i and B.ii also $x_{m} \in L_{\infty}$, and taking into account that $x_{e} \in L_{\infty}$, this implies that $x \in L_{\infty}$. In addition, (35) also implies that $V \in L_{\infty}$. This, together with Lemma 1.i, immediately yields boundedness of all the closed-loop signals.

Remark 3. If $\alpha_{E}=\beta_{E}=0$, then $w, \Gamma_{\alpha}$ and $\Gamma_{\beta}$ have to be redefined as

$$
w(k)=\left[\begin{array}{c}
x(k) \\
r(k)
\end{array}\right], \quad \Gamma_{\alpha}=\alpha \mathbb{I}_{n+1}, \quad \Gamma_{\beta}=\beta \mathbb{I}_{n+1},
$$

with $\mathbb{I}_{n+1}$ standing for the $(n+1) \times(n+1)$ identity matrix.

\subsection{Proof of Corollary 1}

When $A=A(k)$, then $L_{I}^{*}$ and $\phi(k)$ in (24c) and (24b), respectively, become

$$
\begin{aligned}
L_{I}^{*} & =L_{I}^{*}(k)=b^{-1}\left(a_{m, 1}-a_{1}(k), \ldots, a_{m, n}-a_{n}(k), b_{m}, \mathbb{O}_{1, n}\right), \\
\phi(k) & =b\left(L_{I}^{*}(k)-L_{I}(k)\right) .
\end{aligned}
$$

However, (16) allows the corresponding $\Delta \phi(k-1)$ defined in (25) to remain invariant:

$$
\begin{aligned}
\Delta \phi(k-1) & =\phi(k)-\phi(k-1)= \\
& =b\left(L_{I}^{*}(k)-L_{I}^{*}(k-1)\right)-b y_{e}(k) w^{T}(k-1) \Gamma_{\alpha}-b f(k)= \\
& =\left(a_{1}(k-1)-a_{1}(k), \ldots, a_{n}(k-1)-a_{n}(k), \mathbb{O}_{1, n+1}\right)+ \\
& -b y_{e}(k) w^{T}(k-1) \Gamma_{\alpha}-b f(k)= \\
& \approx-b y_{e}(k) w^{T}(k-1) \Gamma_{\alpha}-b f(k) .
\end{aligned}
$$

Hence, the proof follows identically that of Theorem 1 . 


\subsection{Proof of Corollary 2}

The proof follows identically that of Theorem 1 as well after replacing $A_{m}$ by $\mathbb{I}+T_{s} A_{m}$, and $L_{I}^{*}$ by $T_{s} L_{I}^{*}$. This is because, although the discretized plant and reference model matrices are no longer in canonical form, its difference is again a matrix with null elements everywhere but on the last row, exactly as in the original, pure discrete-time case, i.e.

$$
\mathbb{I}+T_{s} A_{m}-\left(\mathbb{I}+T_{s} A\right)=T_{s}\left(A_{m}-A\right) .
$$

Hence, as each column is spanned by $B$, the mismatch can also be tamed by the control action $B u(k)$.

\section{Numerical validation}

The effectiveness of the proposed discrete-time MCS algorithm is shown in this section through a representative numerical example. In particular, the approach will be tested here for a system of the form (1) with

$$
A=\left[\begin{array}{ccc}
0 & 1 & 0 \\
0 & 0 & 1 \\
0.612 & -2.165 & 2.55
\end{array}\right], \quad b=4, \quad x(0)=\left[\begin{array}{c}
0 \\
0.2 \\
-0.3
\end{array}\right]
$$

The reference model is a discrete-time LTI system of the form (3) with

$$
A_{m}=\left[\begin{array}{ccc}
0 & 1 & 0 \\
0 & 0 & 1 \\
0.252 & -1.2 & 1.9
\end{array}\right], \quad b_{m}=1, \quad x_{m}(0)=0,
$$

and the reference input, $r(k)$, is a sinusoidal wave with amplitude 4 and period $2 \pi$.

For the design of the control law we assume that the entries of the plant matrices are unknown but within the following ranges: $-a_{1} \in[-0.148,0.652]$, $-a_{2} \in[-2.2,-0.2],-a_{3} \in[1.3,2.5], b \in[3.36,4.64]$. According to this possible plant parameter variation and in order to satisfy Assumption C.ii, the integral part of the adaptive gains has been limited as follows: $L_{X I_{1}} \in\left[\begin{array}{ll}-0.12 & 0.12\end{array}\right]$, $L_{X I_{2}} \in\left[\begin{array}{ll}-0.3 & 0.3\end{array}\right], L_{X I_{3}} \in\left[\begin{array}{ll}-0.18 & 0.18\end{array}\right], L_{E I_{1}} \in\left[\begin{array}{ll}-3 \cdot 10^{-3} & 3 \cdot 10^{-3}\end{array}\right], L_{E I_{2}} \in$ $\left[\begin{array}{ll}-2 \cdot 10^{-3} & 2 \cdot 10^{-3}\end{array}\right], L_{E I_{3}} \in\left[\begin{array}{ll}-4 \cdot 10^{-3} & 4 \cdot 10^{-3}\end{array}\right]$, and $L_{R I} \in\left[\begin{array}{ll}-0.3 & 0.3\end{array}\right]$. Moreover, $L_{X I}(0)=0, L_{R I}(0)=0$ and $L_{E I}(0)=0$, thus fulfilling requirement (15) in Theorem 1. 


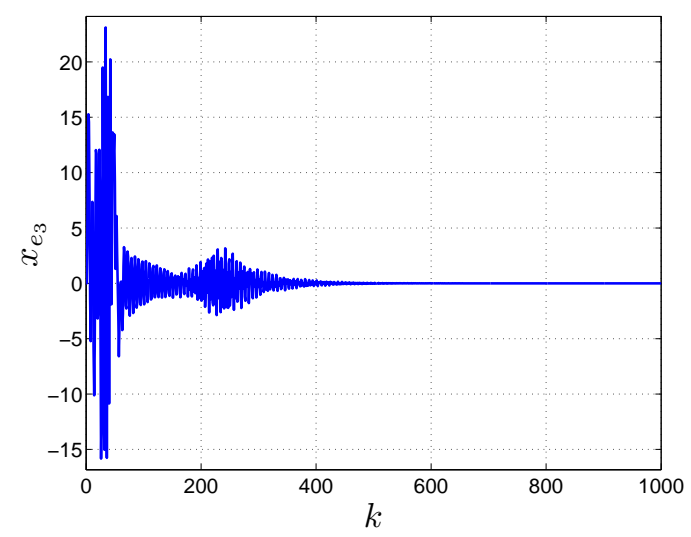

Figure 1: Tracking error evolution of $x_{e_{3}}$.

The adaptive weights are selected as a trade-off between convergence time and reactivity of the control actions. Specifically, we have set $\alpha=10^{-1}$ and $\alpha_{E}=10^{-2}$, while $\beta=\frac{3}{4} \alpha$ and $\beta_{E}=\frac{3}{4} \alpha_{E}$.

Finally, the one-delay issue has been tackled following Remark 2.iv. Thus, we have implemented the simple yet effective estimate of $y_{e}(k+1)$ proposed in $[24,12]$.

\subsection{Performance of the DTMCSI-PP algorithm}

Figure 1 shows the tracking error evolution for the third state variable, $x_{e_{3}}$, and its convergence to zero. Notice that, according to (1) and (36), $x_{e_{1}}(k)=x_{e_{3}}(k-2)$ and $x_{e_{2}}(k)=x_{e_{3}}(k-1)$. Hence, the convergence to zero of $x_{e_{3}}$ guarantees that of the entire tracking error vector.

Figure 2 shows that the integral parts of the adaptive gains, $L_{I}$ in (22a), converge asymptotically to a finite value once the tracking error goes to zero. Notice that upper or lower gain bounds are plotted in the same figure for those adaptive gains which are locked. These limits are depicted as solid lines with the color of the corresponding adaptive gain.

As clearly shown in Figure 2d, the gain $L_{X I_{3}}$ is locked during the transient but it enters again the non-locking region as the tracking error goes to zero. Notice that, as shown in Figure 2c, also $L_{E I_{2}}$ and $L_{E I_{3}}$ are locked in a short time interval, but a zoom is not reported for the sake of brevity.

Figure 2 also confirms the boundedness of the adaptive gains. Indeed, the boundedness of $x_{e}(k)$ (shown in Figure 1) guarantees that of the proportional 
gains, $L_{P}$ in (22b), and consequently the boundedness of $L$ in (21). In addition, as $x_{e} \rightarrow 0$ then $L \rightarrow L_{I}$ as $k \rightarrow+\infty$.

\subsection{Robustness to parameter variations and external $L_{2}$-disturbances}

In order to numerically test the robustness of the control approach, a perturbation in the plant parameters and a square-summable disturbance have also been introduced in the simulation scenario.

Specifically, the behavior in case of a sudden change of the plant parameters values with respect to their nominal ones is investigated. Namely, when $k=1000$, the plant matrix $A$ and the parameter $b$ become

$$
\widetilde{A}=\left[\begin{array}{ccc}
0 & 1 & 0 \\
0 & 0 & 1 \\
0.3 & -0.8 & 1.8
\end{array}\right], \quad \widetilde{b}=3.5,
$$

which fall within the above specified range of variation. Notice also that, differently from $A, \widetilde{A}$ is not Hurwitz, and therefore the plant becomes suddenly unstable.

Furthermore, at the $k=1500$ sample, the $L_{2}$ disturbance shown in Figure 3, which affects the system dynamics as indicated in (1), is activated.

In Figure 4 it is clear that the control algorithm is able to reject such unexpected parameter variations and disturbances and, through adaptation, to achieve again excellent tracking performance. As in the previous case, adaptive gains converge asymptotically to a finite value while the tracking error goes to zero. (Notice that upper or lower gain bounds are reported in Figure 4 for those adaptive gains which are locked. These limits are depicted as solid lines with the color of the corresponding adaptive gain.) In this case, the gain $L_{X I_{3}}$ is locked during the first time instants after the switch of the plant paraments. In addition, the gains $L_{E I_{1}}$, $L_{E I_{2}}$ and $L_{E I_{3}}$ are saturated during the entire activation of the disturbance. Nevertheless, when the unknown external excitation disappears, these gains reenter the non-locking region.

\section{DTMCSI-PP control of an automotive actuator}

A fundamental automotive actuator, the Electronic Throttle Body (ETB), is used here to assess the performance of the novel DTMCSI-PP adaptive algorithm in control engineering applications. 


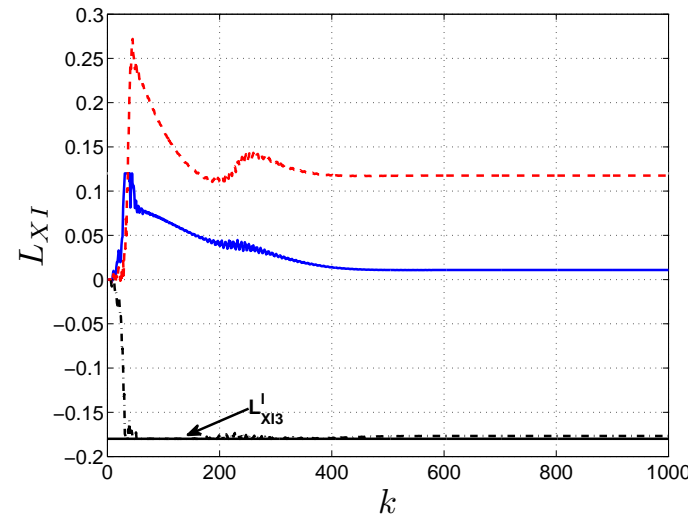

(a)

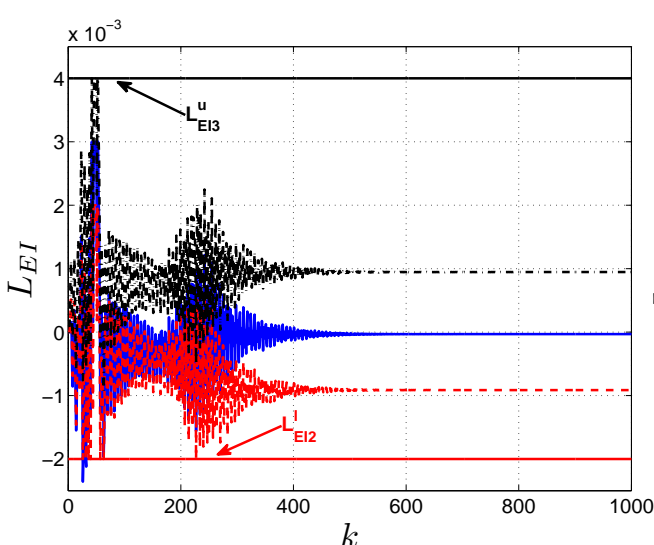

(c)

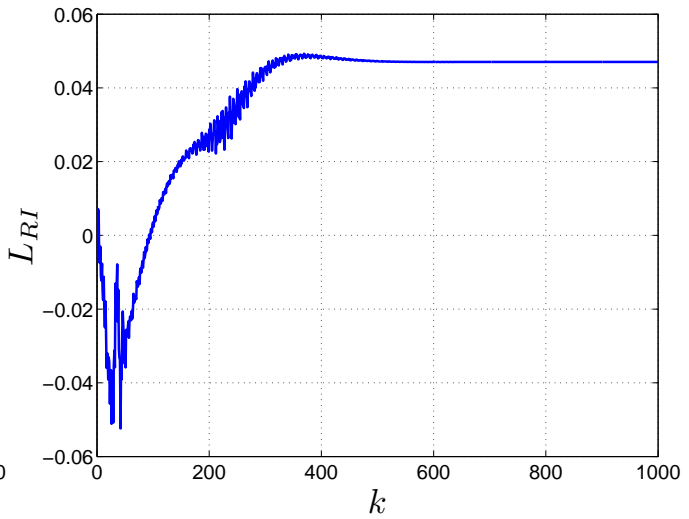

(b)

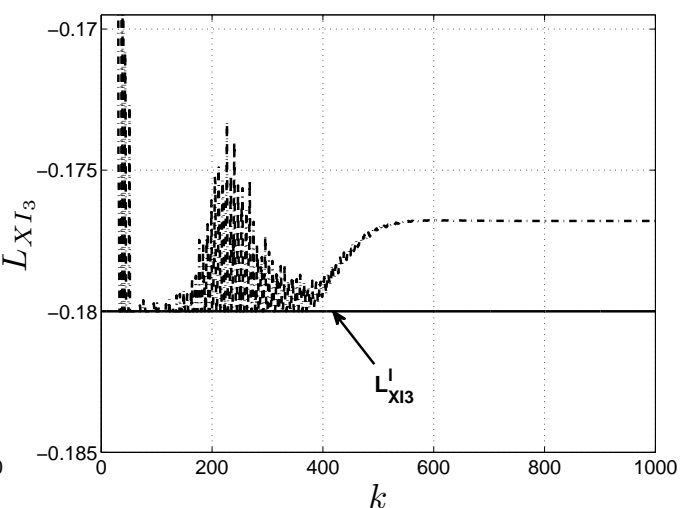

(d)

Figure 2: Adaptive gains. (a) $L_{X I}: L_{X I_{1}}$ (solid blue line), $L_{X I_{2}}$ (dashed red line), $L_{X I_{3}}$ (dashed-dotted black line); (b) $L_{R I}$; (c) $L_{E I}: L_{E I_{1}}$ (solid blue line), $L_{E I_{2}}$ (dashed red line), $L_{E I_{3}}$ (dashed-dotted black line); (d) Detail of the transient of the adaptive gain $L_{X I_{3}}$. Upper or lower gain bounds are reported for those adaptive gains which are locked. These limits are depicted as solid lines with the same color of the corresponding adaptive gain. 


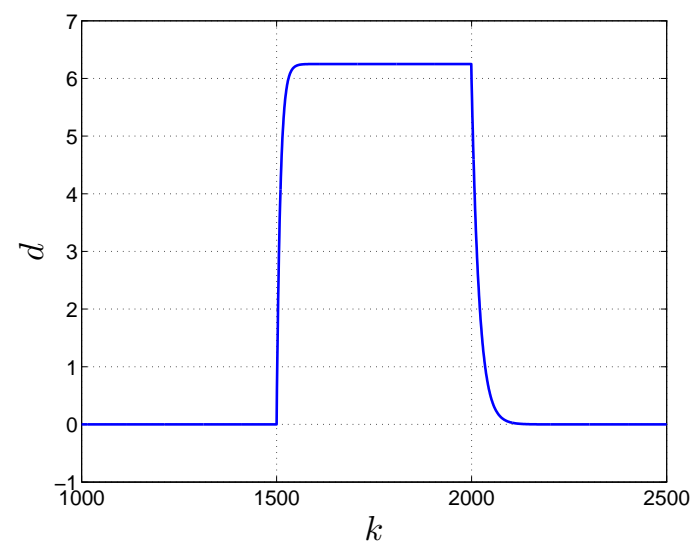

Figure 3: Square-summable disturbance.

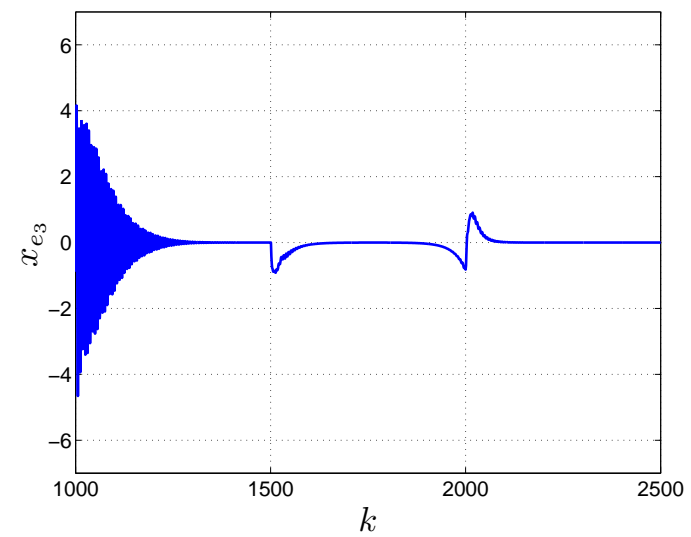

Figure 4: Tracking error evolution of $x_{e_{3}}$ under parameter variations and external disturbances. 


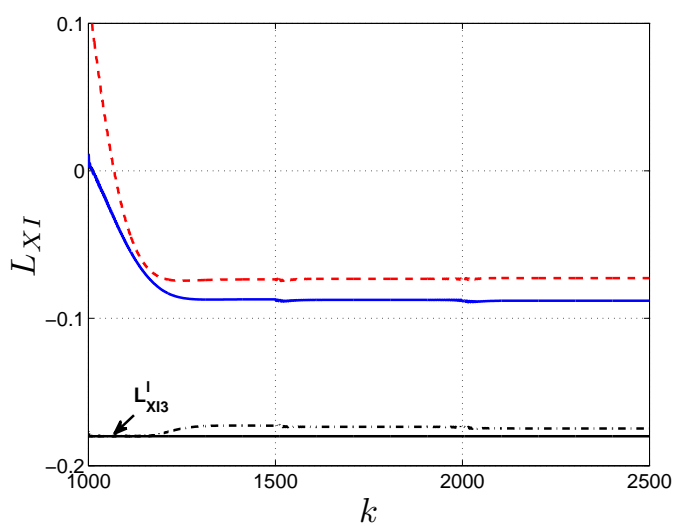

(a)

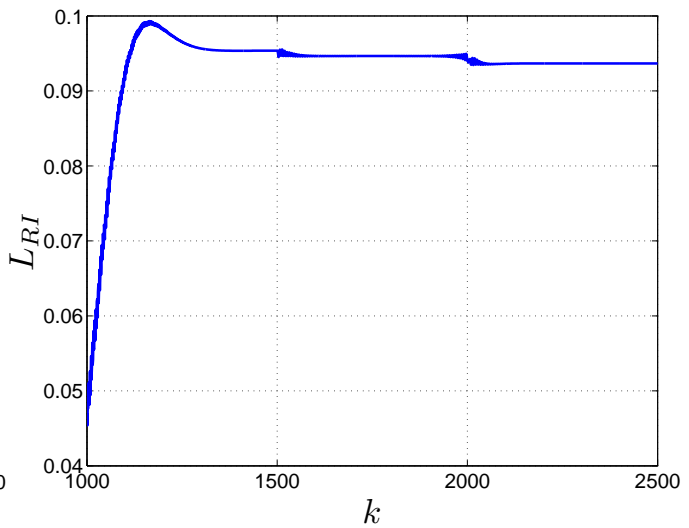

(b)

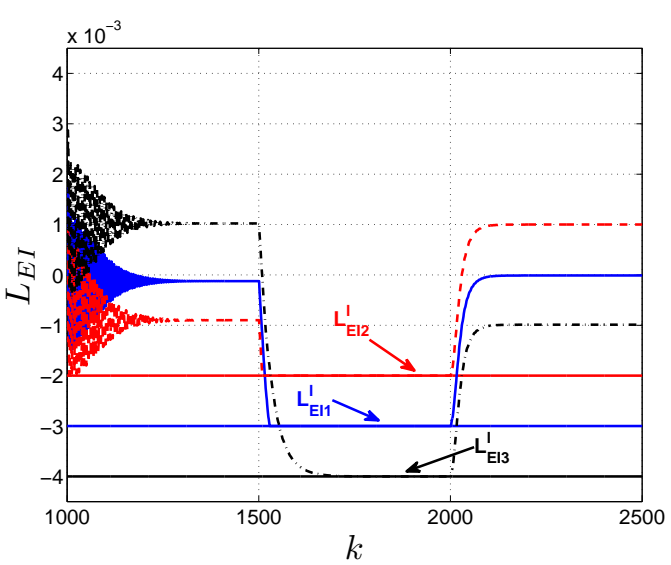

(c)

Figure 5: Adaptive gains under perturbing conditions. (a) $L_{X I}: L_{X I_{1}}$ (solid blue line), $L_{X I_{2}}$ (dashed red line), $L_{X I_{3}}$ (dashed-dotted black line); (b) $L_{R I}$; (c) $L_{E I}$ : $L_{E I_{1}}$ (solid blue line), $L_{E I_{2}}$ (dashed red line), $L_{E I_{3}}$ (dashed-dotted black line). 
The ETB is a mechatronic device that regulates the air flow coming into an internal combustion engine. Hence, controlling precisely its plate position is fundamental to improve performance of higher level engine control strategies, e.g., idle speed control [19] and air-to-fuel ratio control [20], just to name a few. On the other hand, the ETB system is affected by many nonlinearities which can dramatically alter its dynamics such as a piecewise linear restoring torque, friction, impacts and backlash and nonlinear aerodynamic torques. Moreover, manufacturing tolerances, variable operating conditions and mechanical wear often cause uncertainty in system parameter values. The reader is referred to $[30,11]$ for further details.

Due to its challenging control features, the ETB has often been chosen as an ideal case study to investigate the performance and robustness of adaptive control schemes in the face of model uncertainties and disturbances as discussed, for example, in [11], [1], [3], [28], [7], [18]. Furthermore, when MRAC algorithms are used to tame the ETB dynamics, the presence of unmodeled terms can induce an unbounded drift of the adaptive gains [2], which are undesirable for safety reasons.

From a modeling viewpoint, it has been show in [11,27] that, by choosing as state variables the position and velocity of the valve and as control input the armature voltage to the DC motor embedded in the system, the ETB dynamics can be approximated as a second order LTI system in control canonical form subjected to nonlinear disturbances, which is required by the DTMCSI-PP algorithm. For all those reasons, the ETB is an excellent device to test the adaptive law presented in Section 2. The reader is referred to [8] for further details on the ETB.

\subsection{DTMCSI-PP implementation details}

As reference model for the adaptive controller we have selected a second order, continuous-time LTI system in control canonical form with a settling time of about $135 \mathrm{~ms}$, unitary gain, and step response without oscillations. The reference model has been then discretized using a forward Euler's method with sampling period $T=10^{-3} \mathrm{~s}$. This value is in agreement with automotive hardware limitations and yields a stable reference model (3).

The tracking error during tip-in/tip-out conditions has been limited with a Smooth Trajectory Reference (STR) implemented as a first-order filter as in [11, 27].

The one-delay problem has been addressed as in [24]. This approach for solving the one-delay problem has also been validated experimentally in $[8,27]$ for 
the discrete-time MCS control of an ETB. The numerical results reported in next subsection confirm again the effectiveness of this choice.

It is worth remarking that the velocity of the plate is not available to the controller. In accordance with $[11,8,27]$, in order to not complicate the control architecture with the use of observer [42] or to introduce noise and delays in the control system with the implementation of a derivative filter, here we implement the DTMCSI-PP algorithm using only position measurements. In so doing, we provide an additional evidence of the robustness and ease of implementation of MCS-based control strategies.

The bounds of the integral part of the adaptive gains have been selected in accordance with the expected variation of the ETB plant parameters, namely: $L_{X I_{1}}$ $\in[-0.250 .25], L_{E I_{1}} \in[-0.150 .15], L_{R I} \in[-0.250 .25]$. The adaptive weights have been selected heuristically as usual, i.e., as a trade-off between convergence time and control reactivity. Here we chose $\alpha=8 \cdot 10^{-5}, \alpha_{E}=8 \cdot 10^{-6}, \beta=\frac{3 \alpha}{4}$ and $\beta_{E}=\frac{3 \alpha_{E}}{4}$.

Finally, we point out that the numerical analysis has been carried out using an accurate model of the ETB, which was experimentally validated in [10].

\subsection{Numerical results}

In this subsection we assess the performance of the novel adaptive algorithm in taming the ETB dynamics. We first consider as reference signal a sinusoidal wave with amplitude $35 \mathrm{deg}$, bias $50 \mathrm{deg}$, and period $4 \mathrm{~s}$. In order to test the controller in a more realistic scenario, we have introduced an additional external sinusoidal torque with amplitude $0.1 \mathrm{Nm}$ and period $4 \mathrm{~s}$ in the ETB dynamics to emulate the aerodynamic torque. Furthermore, aiming at simulating the noise of the resistive potentiometers used to measure the valve opening, a white noise with variance 0.4 has been added to the plate position available to the controller.

The integral part of the adaptive control gains evolve with time as depicted in Figure 6c, causing an increasingly better tracking of the reference trajectory. This is evident by analyzing the tracking performance in Figures $6 \mathrm{a}$ and $6 \mathrm{~b}$, while the feasibility of the control action is shown in Figure 6d. We note that for the ETB control the persistent disturbance acting on the plant dynamics is bounded but it may induce instability due to the drifting on the adaptive control gains [2]. However, the parameter projection-based gain locking strategy prevents the onset of such an undesirable phenomenon, with some adaptive control gains being periodically locked over the manoeuvre. Precisely, $L_{X I}$ is locked at its lower value, while $L_{E I}$ is saturated periodically to its upper bound. Notice these bounds are 


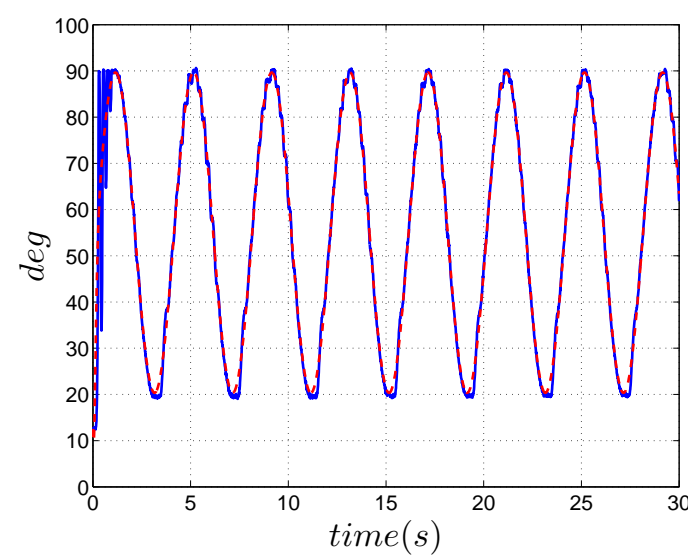

(a)

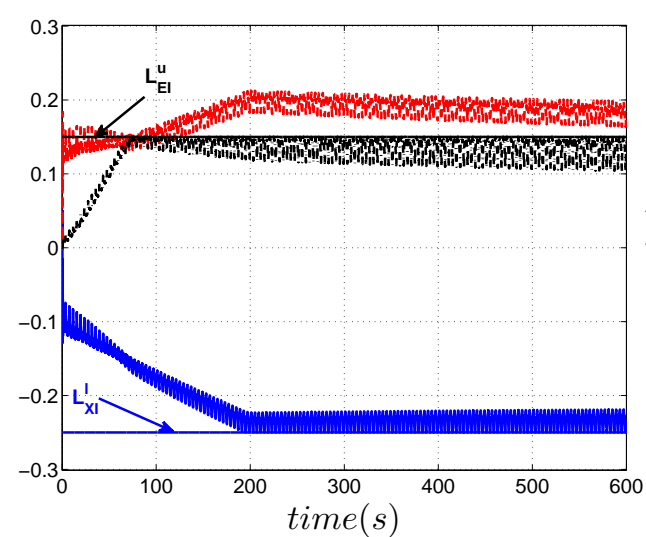

(c)

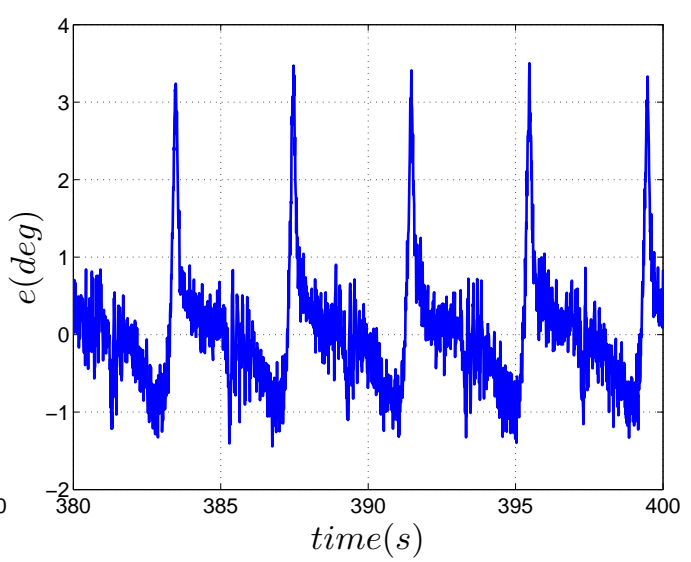

(b)

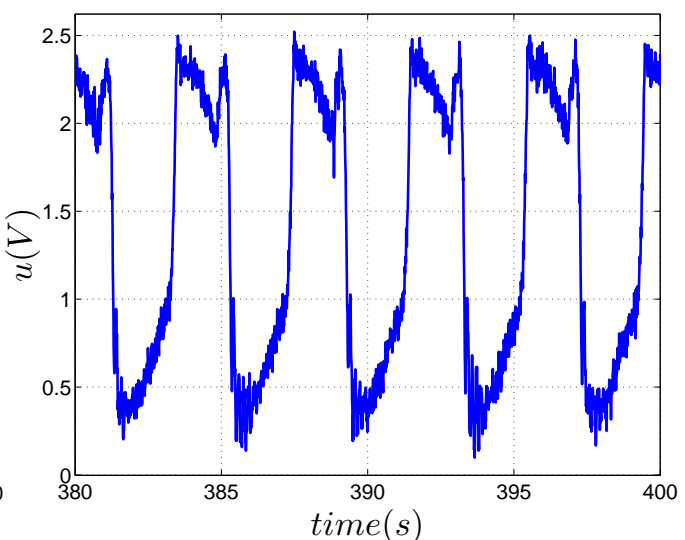

(d)

Figure 6: ETB control performance. (a) Tracking performance: plate position (blue solid line), reference model position (dashed red line). (b) Residual tracking error in steady state. (c) Control gains: $L_{X I_{1}}$ (solid blue line), $L_{R I}$ (dashed red line), $L_{E I_{1}}$ (dashed-dotted black line). (d) Control action in steady state. 
also reported in Figure $6 \mathrm{c}$ as solid lines with the color of the corresponding adaptive gain.

As in the case of the numerical example in Section 4, the boundedness of the integral gains $6 \mathrm{c}$ imply the boundedness of the adaptive control gains.

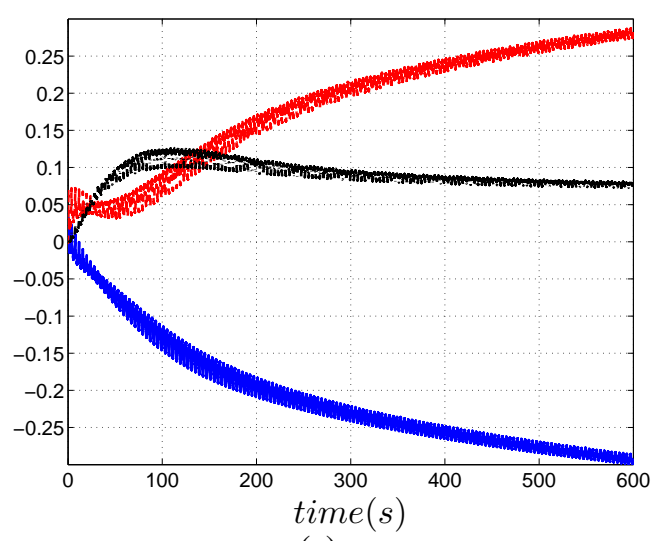

(a)

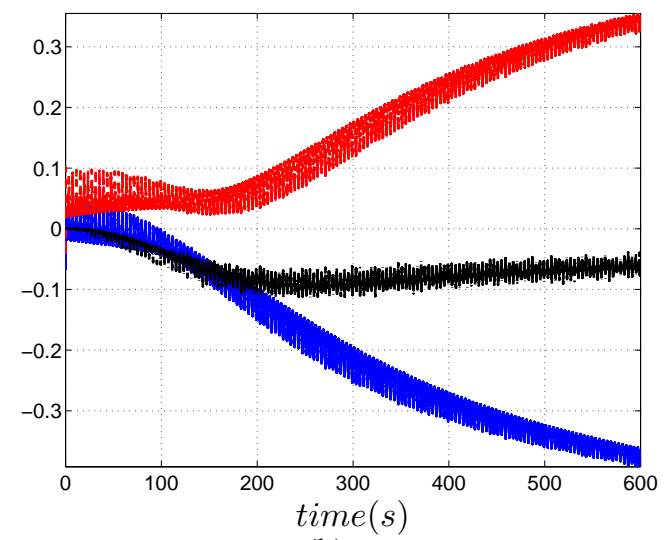

(b)

Figure 7: Adaptive control gains. (a) DTMCSI algorithm. $L_{X I_{1}}$ (solid blue line), $L_{R I}$ (dashed red line), $L_{E I_{1}}$ (dashed-dotted black line). (b) MCSI algorithm. $L_{X I_{1}}$ (solid blue line), $L_{R I}$ (dashed red line), $L_{E I_{1}}$ (dashed-dotted black line).

We stress that boundedness of the control gains is not assured by MCS algorithms available in the literature when unmodelled dynamics and persistent disturbances affect plant dynamics. To better appreciate this feature of the novel control scheme, we have implemented for the ETB system the MCSI - discretized via Tustin method - and DTMCSI algorithms, which have been presented in $[37,11]$ and [26], respectively. We point out that, under the same working conditions, these controllers provide a residual error which is comparable to that in Figure $6 \mathrm{~b}$, but Figure 7 clearly shows that the integral part of their control gains diverges.

In order to confirm that the DTMCSI-PP strategy provides tracking performances similar or better than those achievable by other MCS strategies, namely MCSI and DTMCSI algorithms, the control performances provided by these three controllers are compared when the reference input is the long reference manoeuvre composed by the signals listed in Table 1, which is similar to that used in [26]. The reference signal has been split into six relevant subsets so as to better quantify the tracking capabilities. The first four of them are already indicated in Table 1: learning (S1), after learning (S2), miscellaneous (S3), and sequence of 
Table 1: Reference model input

\begin{tabular}{|c|c|c|c|c|c|c|}
\hline Signal set & Signal & Type & Amplitude (deg) & Bias (deg) & Period (s) & Duration (s) \\
\hline \hline & 1 & Constant & 20 & & & 10 \\
\hline S1: Learning & 2 & Square wave & 25 & 45 & 6 & 60 \\
& 3 & Sinusoidal wave & 25 & 45 & 6 & 60 \\
& 4 & Square wave & 25 & 55 & 6 & 30 \\
& 5 & Sinusoidal wave & 25 & 45 & 6 & 30 \\
& 6 & Square wave & 10 & 50 & 6 & 30 \\
& 7 & Sinusoidal wave & 10 & 50 & 6 & 30 \\
& 8 & Constant & 40 & - & - & 10 \\
\hline S2: After learning & 9 & Square wave & 25 & 45 & 6 & 60 \\
& 10 & Sinusoidal wave & 25 & 45 & 6 & 60 \\
& 11 & Square wave & 25 & 55 & 6 & 30 \\
& 12 & Sinusoidal wave & 25 & 55 & 6 & 30 \\
& 13 & Square wave & 10 & 50 & 6 & 30 \\
& 14 & Sinusoidal wave & 10 & 50 & 6 & 30 \\
& 15 & Constant & 40 & - & - & 10 \\
\hline S3: Miscellaneous & 16 & Square wave & 25 & 55 & 3 & 30 \\
& 17 & Square wave & 25 & 55 & 2 & 10 \\
& 18 & Sinusoidal wave & 25 & 55 & 3 & 30 \\
& 19 & Sinusoidal wave & 25 & 55 & 1 & 5 \\
& 20 & Square wave & 35 & 55 & 5 & 30 \\
& 21 & Square wave & 35 & 55 & 3 & 10 \\
& 22 & Sinusoidal wave & 35 & 55 & 5 & 30 \\
& 23 & Sinusoidal wave & 35 & 55 & 1 & 5 \\
& 24 & Square wave & 7.5 & 52.5 & 5 & 20 \\
& 25 & Sinusoidal wave & 10 & 50 & 4 & 20 \\
\hline S4: Sequence of steps & 26 & Sequence of steps & 15 (step amplitude) & 15 (initial value) & - & 120 \\
& 27 & Sequence of steps & 5 (step amplitude) & 10 (initial value) & - & 380 \\
\hline
\end{tabular}

steps (S4). The remaining two are: square waves (S5), i.e., all the square waves of the manoeuvre, and sinusoidal waves (S6), i.e., all the sinusoidal waves of the manoeuvre.

The tracking performance of each controller is evaluated computing the maximum absolute percentage tracking error over each set. Figure 8a shows that the novel approach always provides better tracking when compared to the MCSI algorithm, while the presence of the locking strategy makes the control performance of the DTMCSI-PP algorithm slightly worse than that provided by the DTMCSI controller on sets S1, S2, S5 and S6. This slight loss of performance is acceptable taking into account that, for a similar manoeuvre, both the DTMCSI and the MCSI algorithm show diverging control gains, while the adaptive gains of the DTMCSIPP algorithm remain bounded over the entire manoeuvre (see Figure 8b). For this case, each adaptive gain is saturated in some time interval. Specifically, $L_{X I}$ is locked at its lower value at certain time instants, while $L_{E I}$ and $L_{E I}$ at their upper bounds. Notice these bounds are also reported in Figure $8 \mathrm{~b}$ as solid lines with the color of the corresponding adaptive gain. 


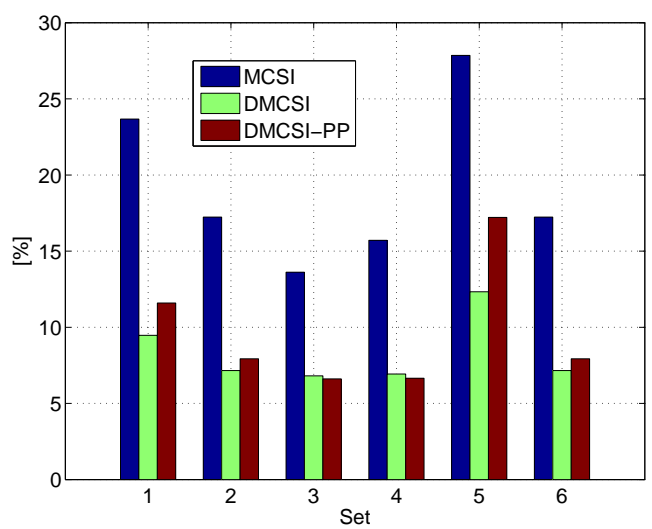

(a)

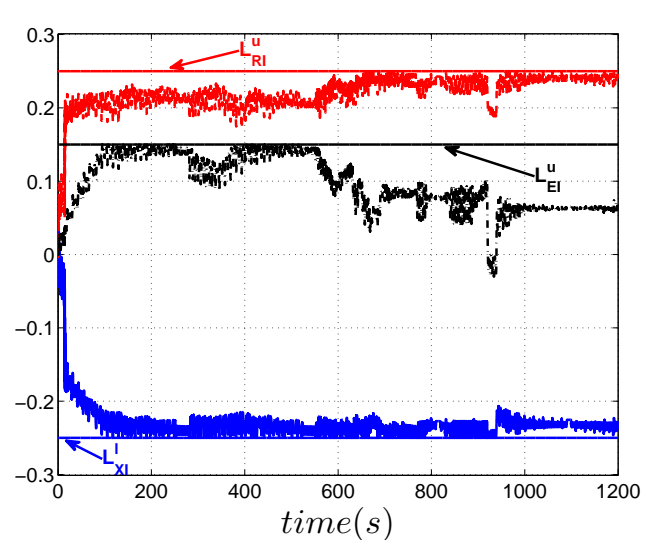

(b)

Figure 8: Adaptive control gains. (a) Maximum absolute percentage tracking error. (b) Integral adaptive gains of the novel controller when the reference input is composed by the signals listed in Table $1 . L_{X I_{1}}$ (solid blue line), $L_{R I}$ (dashed red line), $L_{E I_{1}}$ (dashed-dotted black line).

\section{Conclusions}

In this paper the problem of diverging adaptive gains for discrete-time MCS algorithms has been tackled with a parameter projection method. This locking strategy has been here exploited to prevent critical closed-loop behavior, i.e., possible instability of discrete-time MCS controlled plants caused by a drifting of the control gains when nonlinear perturbations and disturbances affect the plant dynamics.

The parameter projection method has been added to a pre-existing MCS algorithm. A consistent proof of stability of the overall closed-loop system with convergence to zero of the tracking error has been carried out using a discrete-time Lyapunov approach. The only extra assumption with respect to other adaptive algorithms belonging to the same family is that the novel MCS strategy requires knowledge of the range of variation of each plant parameter. This assumption is not as restrictive as it might appear at first, as for many engineering problems some knowledge of the plant parameters is often available.

Furthermore, it has been explicitly proven that the resulting algorithm is robust with respect to $L_{2}$ disturbances and, under further assumptions that are typical for MCS control schemes, the control action can be successfully applied to discretetime LTV plants and continuous-time LTI systems. 
The effectiveness of the novel control scheme has been numerically tested on a set of relevant examples including the control of a nonlinear automotive actuator. Comparison with pre-existing MCS algorithms have proven the effectiveness of the novel strategy to keep the adaptive gains bounded for this practical case study.

\section{Acknowledgements}

J.M. Olm was partially supported by the spanish Ministerio de Educación project DPI2013-41224-P and the catalan AGAUR project 2014 SGR 267.

\section{References}

[1] B. Alt, J.P. Blath, F. Svaricek, and M. Schultalbers. Self-tuning control design strategy for an electronic throttle with experimental robustness analysis. In Proceedings of the American Control Conference, pages 6127-6132, Baltimore, ML, July 2010.

[2] B.D.O. Anderson, R.R. Bitmead, C.R. Johnson, P.V. Kokotovic, R.L. Kosut, I.M.Y. Mareels, L. Praly, and B.D. Riedle. Stability of adaptive systems: passivity and averaging analysis. The M.I.T. Press, Cambridge, MA and London, 1986.

[3] M. Barić, I. Petrović, and N. Perić. Neural network-based sliding mode control of electronic throttle. Engineering Applications of Artificial Intelligence, 18(8):951-961, 2005.

[4] H. Benchoubane and D.P. Stoten. The decentralized minimal controller synthesis algorithm. International Journal of Control, 56(4):967-983, 1992.

[5] O.S. Bursi, D.P. Stoten, and L. Vulcan. Convergence and frequency-domain analysis of a discrete first-order model reference adaptive controller. Structural Control and Health Monitoring, 14(5):777-807, 2007.

[6] F. Chen, F. Lu, B. Jiang, and G. Tao. Adaptive compensation control of the quadrotor helicopter using quantum information technology and disturbance observer. Journal of the Franklin Institute, 351(1):442-455, 2014. 
[7] M. Corno, M. Tanelli, S.M. Savaresi, and L. Fabbri. Design and validation of a gain-scheduled controller for the electronic throttle body in ride-bywire racing motorcycles. IEEE Transactions on Control Systems Technology, 19(11):18-30, 2011.

[8] M. di Bernardo, A. di Gaeta, U. Montanaro, J. M. Olm, and S. Santini. Experimental validation of the discrete-time MCS adaptive strategy. Control Engineering Practice, 21(6):847-859, 2013.

[9] M. di Bernardo, A. di Gaeta, U. Montanaro, J.M. Olm, and S. Santini. Discrete-time mrac with minimal controller synthesis of an electronic throttle body. In 18th IFAC World Congress, pages 5064-5069, Aug. 2011.

[10] M. di Bernardo, A. di Gaeta, U. Montanaro, and S. Santini. A comparative study of the new LQ-MCS control on an automotive electro-mechanical system. In IEEE International Symposium on Circuits and Systems, pages 552-555, Sep. 2008.

[11] M. di Bernardo, A. di Gaeta, U. Montanaro, and S. Santini. Synthesis and experimental validation of the novel LQ-NEMCSI adaptive strategy on an electronic throttle valve. IEEE Transactions on Control Systems Technology, 18(6):1325-1337, 2010.

[12] M. di Bernardo, F. di Gennaro, J. M. Olm, and S. Santini. Discrete-time minimal control synthesis adaptive algorithm. International Journal of Control, 83(4):2641-2657, 2010.

[13] M. di Bernardo, U. Montanaro, J.M. Olm, and S. Santini. Model reference adaptive control of discrete-time piecewise linear systems. International Journal of Robust and Nonlinear Control, 23(7):709-730, 2013.

[14] M. di Bernardo, U. Montanaro, and S. Santini. Minimal control synthesis adaptive control of continuous bimodal piecewise affine systems. SIAM Journal on Control and Optimization, 48(7):4242-4261, 2010.

[15] M. di Bernardo, U. Montanaro, and S. Santini. Hybrid model reference adaptive control of piecewise affine systems. IEEE Transactions on Automatic Control, 58(2):304-316, 2013.

[16] M. di Bernardo, C. I. Hoyos Velasco, U. Montanaro, and S. Santini. Experimental implementation and validation of a novel minimal control synthesis 
adaptive controller for continuous bimodal piecewise affine systems. Control Engineering Practice, 20(3):269-281, Mar. 2012.

[17] A. di Gaeta, C. I. Hoyos-Velasco, and U. Montanaro. Cycle-by-cycle adaptive force compensation for the soft-landing control of an electromechanical engine valve actuator. Asian Journal of Control, July 2015. DOI 10.1002/asjc.988.

[18] A. di Gaeta and U. Montanaro. Application of a robust model reference adaptive control algorithm to a nonlinear automotive actuator. International Journal of Automation and Computing, 11(4):377-391, Aug. 2014.

[19] A. di Gaeta, U. Montanaro, and V. Giglio. Idle speed control of GDI-SI engines via ECU-1D engine co-simulation. SAE Technical Paper, 2010-012220, 2010.

[20] A. di Gaeta, U. Montanaro, and V. Giglio. Model-based control of the air fuel ratio for gasoline direct injection engines via advanced co-simulation: An approach to reduce the development cycle of engine control systems. Journal of Dynamic Systems, Measurement, and Control, 133(6):061006(1) -061006(17), 2011.

[21] A.O. Gizatullin and K.A. Edge. Adaptive control for a multi-axis hydraulic test rig. Proceedings of the Institution of Mechanical Engineers - Part I: Journal of Systems and Control Engineering, 221(2):183-198, 2007.

[22] A.J. Hillis, A.J.L. Harrison, and D.P. Stoten. A comparison of two adaptive algorithms for the control of active engine mounts. Journal of Sound and Vibration, 286(1-2):37-54, 2005.

[23] P. Ioannou and B. Fidan. Adaptive Control Tutorial. SIAM, 2006.

[24] I. D. Landau. Adaptive Control. The Model Reference Approach. Marcel Dekker, 1979.

[25] U. Montanaro, A. di Gaeta, and V. Giglio. An mrac approach for tracking and ripple attenuation of the common rail pressure for gdi engines. In 18th IFAC World Congress, pages 4173-4180, Aug. 2011.

[26] U. Montanaro, A. di Gaeta, and V. Giglio. Adaptive tracking control of a common rail injection system for gasoline engines: a discrete-time integral 
minimal control synthesis approach. IEEE Transactions on Control Systems Technology, 21(5):1940-1948, 2013.

[27] U. Montanaro, A. di Gaeta, and V. Giglio. Robust discrete-time MRAC with minimal controller synthesis of an electronic throttle body. IEEE/ASME Transactions on Mechatronics, 19(2):524 - 537, 2014.

[28] D. Pavković, J. Deur, M. Jansz, and N. Perić. Adaptive control of automotive electronic throttle. Control Engineering Practice, 14(2):121-136, 2006.

[29] K. Queiroz, S. Dias, and A. Araujo. Modular variable structure adaptive backstepping controller: Design and stability analysisoriginal research article. Journal of the Franklin Institute, 351(4):2089-2106, 2014.

[30] C. Rossi, A. Tilli, and A. Tonielli. Robust control of a throttle body for drive by wire operation of automotive engines. IEEE Transactions on Control Systems Technology, 8(6):993-1002, 2000.

[31] L. Rossi, A. Irace, U. Montanaro, M. di Bernardo, and G. Breglio. Structural vibration control of a cantilever beam by MRAC method. In Proceedings of the 2nd International Symposium on Reliability of Optoelectronics For Space, Cagliari, Italy, Apr. 2010.

[32] Q. Sang and G. Tao. Adaptive control of piecewise linear systems with applications to NASA GTM. In Proceedings of the American Control Conference, pages 1157-1162, San Francisco, CA, July 2011.

[33] Q. Sang and G. Tao. Adaptive control of piecewise linear systems: the state tracking case. IEEE Transactions on Automatic Control, 57(2):522-528, 2012.

[34] S.E.M. Sebusang and D.P. Stoten. Controller gain bounding in the minimal control synthesis algorithm. In Proceedings of the 30th Southeastern Symposium on System Theory, pages 141-145, Morgantown, WV, March 1998.

[35] D. P. Stoten and H. Benchoubane. Empirical studies of an MRAC algorithm with minimal controller synthesis. International Journal of Control, 51(4):823-849, 1990.

[36] D. P. Stoten and H. Benchoubane. Robustness of minimal controller synthesis algorithm. International Journal of Control, 51(4):851-861, 1990. 
[37] D. P. Stoten and S. A. Neild. The error-based minimal control synthesis algorithm with integral action. Proceedings of the Institution of Mechanical Engineers - Part I: Journal of Systems and Control Engineering, 217(3):187201, 2003.

[38] D.P. Stoten and H. Benchoubane. The extended minimal controller sysnthesis algorithm. International Journal of Control, 56(5):1139 - 1165, 1992.

[39] D.P. Stoten and M. di Bernardo. Application of the minimal control synthesis algorithm to the control and synchronization of chaotic systems. International Journal of Control, 65(6):925-938, 1996.

[40] D.P. Stoten and E.G. Gómez. Adaptive control of shaking tables using the minimal controller synthesis algorithm. Philosophical Transaction of the Royal Society of London, 357(9):1697-1723, 2001.

[41] G. Tao. Adaptive Control Design and Analysis. John Wiley \& Sons, Inc. Hoboken, NJ, 2003.

[42] M. Vasak, I. Petrovic, and N. Peric. State estimation of an electronic throttle body. In Proceedings of the IEEE International Conference on Industrial Technology, volume 1, pages 472-477, 2003.

[43] S. J. Yoo. Adaptive output-feedback control for nonlinear time-delay systems in pure-feedback. Journal of the Franklin Institute, 351(7):3899-3913, 2014. 